\title{
Sand Ripples Induced by Water Shear Flow in an Annular Channel
}

\author{
A. Betat, V. Frette, and I. Rehberg \\ Institut für Experimentelle Physik, Otto-von-Guericke-Universität Magdeburg, Postfach 4120, D-39016 Magdeburg, Germany
}

(Received 27 January 1999)

\begin{abstract}
We have studied experimentally the formation and evolution of sand ripples under water shear flow in a narrow annular channel. The amplitude of the sediment ripples vanishes at a critical value of the shear rate. Near this onset, a band of Fourier modes can be described by exponential growth functions. The growth rates vary linearly with the shear rate. On longer times the sand ripples display complex dynamics.
\end{abstract}

PACS numbers: 47.54. $+\mathrm{r}, 45.70 .-\mathrm{n}, 92.10 . \mathrm{Wa}$

One of the most conspicuous examples of pattern formation in nature is the ripples and dunes formed in sand, either by wind, flowing water, or surface waves on water $[1,2]$. In particular, ripple formation induced by water shear flow is well known: everybody is familiar with ripples in mudflats or on riverbeds. To describe pattern-forming systems like these near onset the normal procedure is to linearize the governing equations [3]. However, for sand there are no well-established equations to start from. In order to make progress, one has to rely on experiments carried out near the onset of pattern formation.

To evade the lack of fundamental equations for sand, two main approaches have been followed in theoretical work. The first consists of various continuum descriptions, which can be valid only on length scales significantly larger than the grain size. Under some model assumptions it has then been possible to treat ripple formation driven by water shear flow in terms of linear stability theory [4-8]. In the second approach, one attempts to model explicitly the grain-level events: the formation and motion of ripples or dunes result from grains that are set in motion by the water, follow some trajectories, and come to rest further downstream. To mimic this motion, cellular automata are employed, which seem to catch many of the qualitative features of bedform formation [9-11].

Most experimental studies have focused on questions of importance in engineering $[12,13]$ or on the longtime behavior [14]. In Refs. [15,16], the early stages of ripple formation were considered. They determined the most unstable mode, and the ripple length was found to be consistent with estimates from linear stability analysis [7]. However, by merely determining the most unstable mode, one cannot deduce whether linearized theories apply.

Furthermore, all these experiments were performed in long and wide rectangular channels. A serious limitation of these setups is that the system must be fed with both water and sand. This might disturb the ripple dynamics, especially close to the threshold where the system is very susceptible.

We have built an experimental setup that has several advantages compared to previous work: strict mass conservation, rotational symmetry that allows unrestricted use of Fourier techniques, quasi-one-dimensional geometry, as well as high spatial and temporal resolution. We find that a linear description indeed is possible near onset. While the ripple formation in this respect resembles other pattern-forming systems, there are also striking differences that originate from the unusual properties of the sand: the ripple pattern does not disappear as the driving is reduced, and more complex behavior arises when the amplitude is comparable to the grain size. Finally, we find a series of intriguing features in the dynamics as the ripples are driven into the strongly nonlinear regime.

Our experimental setup [see Fig. 1(a)] consists of a $15 \mathrm{~mm}$ wide and $58 \mathrm{~mm}$ high annular channel of $292 \mathrm{~mm}$ diameter formed by a solid inner cylinder and an outer cylinder. The channel is filled with glass beads 280$300 \mu \mathrm{m}$ in diameter to a height of $38 \mathrm{~mm}$. It is lowered into an aquarium that is filled with water to a height of $14 \mathrm{~cm}$. The shear flow is generated with a rotor disc that extends $4 \mathrm{~mm}$ from the top into the channel. The typical roughness of the sand surface before the start of a run is about $3 d$, where $d=290 \mu \mathrm{m}$ is the mean grain diameter. Six cameras, controlled by a single frame grabber card,

(a)

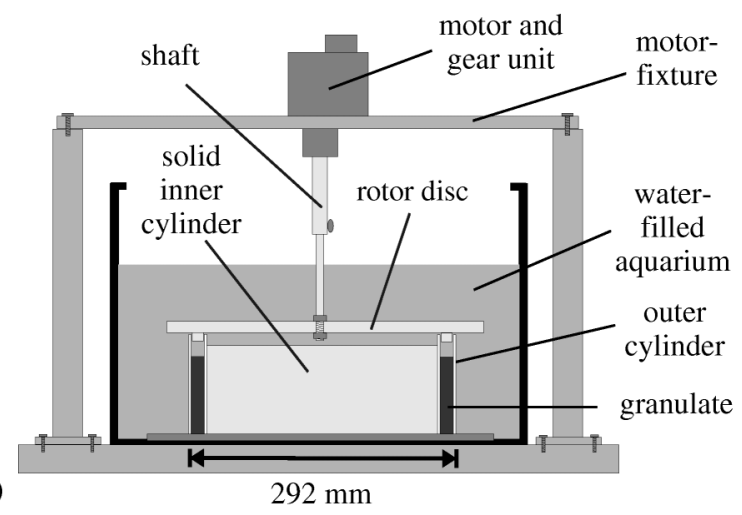

(b) $292 \mathrm{~mm}$

FIG. 1. (a) Side view of the experimental setup; (b) the six camera images of the sand-water interface are separated by vertical white lines. The images have been expanded in the vertical direction. 
are placed at angular distances of $60^{\circ}$ and cover the entire circumference of the channel. The spatial resolution is about $0.7 d$.

At a constant sampling rate $f_{s}=1 / T_{s}$ the images of all six cameras are stored. The boundary between bright sand and darker water is detected, rescaled to account for the geometrical aberration, and the six pieces are matched together. An example is shown in Fig. 1(b). The link between two images is indicated by white vertical lines. The water is flowing from the left to the right.

The boundary curve $h\left(x, n T_{s}\right)$, where $x$ is the spatial position and $n T_{s}$ with $n \in \mathbb{N}$ the elapsed time, is Fourier decomposed,

$$
\begin{aligned}
H\left(x, n T_{s}\right)=\frac{a_{0}}{2}+\sum_{m=1}^{N}\{ & \left\{a_{m}\left(n T_{s}\right) \cos \left(\frac{m 2 \pi x}{L}\right)\right. \\
& \left.+b_{m}\left(n T_{s}\right) \sin \left(\frac{m 2 \pi x}{L}\right)\right\},
\end{aligned}
$$

where $L$ is the circumference of the channel and $2 N=$ 4112 the number of data points in the boundary curve. The mean ripple amplitude $A_{a v}$ is given by the square root of the sum of all Fourier amplitudes as $A_{a v}=$ $\sqrt{\sum_{m=1}^{N}\left(a_{m}^{2}+b_{m}^{2}\right)}$.

Following earlier work on bedform formation (see, e.g., Refs. $[15,17])$ we quantify the applied shear stress by the particle Reynolds number $\mathrm{Re}_{*}=d u_{\tau} / \nu$. This implies that the relevant length scale is given by the mean grain diameter $d, \nu$ is the kinematic viscosity of water at room temperature, and $u_{\tau}=\left(\nu \frac{\partial U}{\partial y}\right)^{1 / 2}$ is the unperturbed shear velocity acting on the particles. $\frac{\partial U}{\partial y}$ was estimated by assuming a linear profile for the fluid velocity $U(y)$ in the middle of the channel.

The left-hand part of Fig. 2 shows the typical longtime evolution of the boundary curve. The abscissa gives the position along the channel. In the right-hand part of Fig. 2 the first 25 Fourier intensities $\left(a_{m}^{2}+b_{m}^{2}\right)$ are plotted. On the horizontal axis of the right-hand part the modes of the Fourier series are given. The shear stress was $\operatorname{Re}_{*}=2.40$. The sampling time was $T_{s}=400 \mathrm{~s}$, but only every eighth curve is plotted. Between two consecutive boundary curves an offset of $8 \mathrm{~mm}$ is added; the offset between two consecutive spectra is $2.5 \mathrm{~mm}^{2}$. After the nucleation of one ripple at a specific position there is a fast generation of more ripples downstream of the first one. This rather rapid dynamics can be observed until the whole system is filled with ripples. After about $6 \mathrm{~h}$ the pattern reaches an "equilibrium state" with a rather narrow spectrum. The ripples drift with a velocity of approximately $9 \mathrm{~cm} / \mathrm{d}$, corresponding to an oscillation period of $27 \mathrm{~h}$, in the downstream direction. As is shown in Fig. 3, the mean ripple amplitude saturates upon reaching this state. Interesting and worth noting is the breakup of a seemingly "stable" state after an elapsed time of about $50 \mathrm{~h}$ as can be seen in Fig. 2(b): $m=9$ as the dominant mode splits into $m=10$ and $m=6$. As shown in the inset of

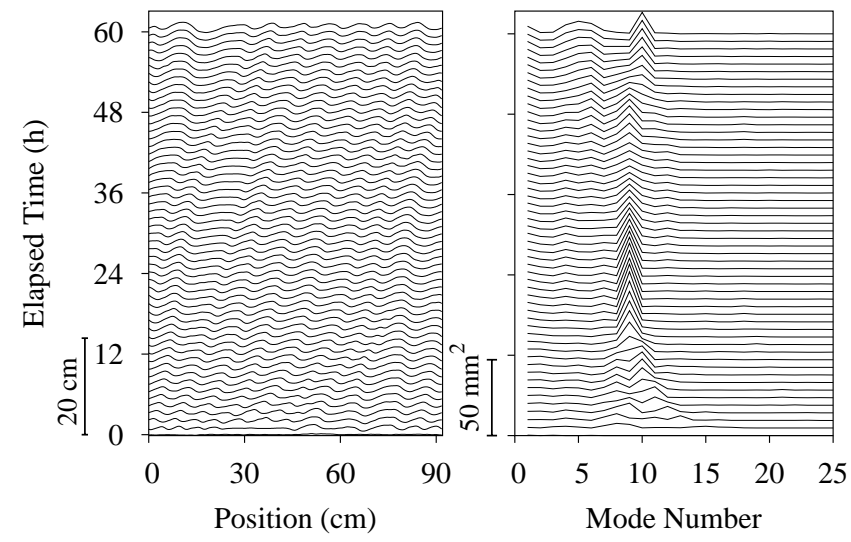

FIG. 2. Temporal evolution of the sand-water interface along the circumference of the channel (left) and of the intensities of the first 25 modes of the Fourier series (right) for $\mathrm{Re}_{*}=2.40$. Consecutive data sets are separated $3200 \mathrm{~s}$ in time and are plotted above each other with a certain offset. The scale is given at the left side of each part of the figure.

Fig. 3, a relatively rapid change in the mean amplitude is associated with this event. The observation of an initial regime of rapid growth followed by a saturation of the mean amplitude is in qualitative agreement with previous work $[14,15]$. The time needed to reach the equilibrium state becomes progressively longer as the shear rate is reduced towards threshold.

To get deeper insight into the transient state before the mean amplitude reaches the plateau we reduced the sampling time to $T_{s}=14 \mathrm{~s}$, the highest temporal resolution we are able to achieve. The early evolution of the amplitudes of three different Fourier modes is shown in Fig. 4. The experiment was performed at $\mathrm{Re}_{*}=2.40$, the same shear stress as for the experiment in Fig. 2. Figures 4(a) and 4(b) correspond to modes in the vicinity of the fastest growing mode, which was determined to be $m=10$, corresponding to a wave number $k=0.69 \mathrm{~cm}^{-1}$ (see Fig. 5

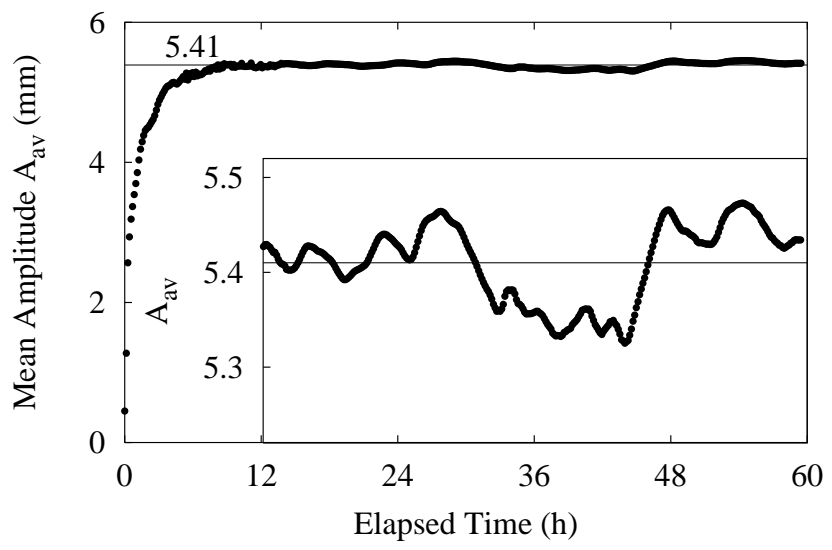

FIG. 3. Evolution of the mean amplitude of the sand-water interface for $\mathrm{Re}_{*}=2.40$. The inset is a partial enlargement. The solid line denotes the mean value obtained by neglecting the first $12 \mathrm{~h}$. 

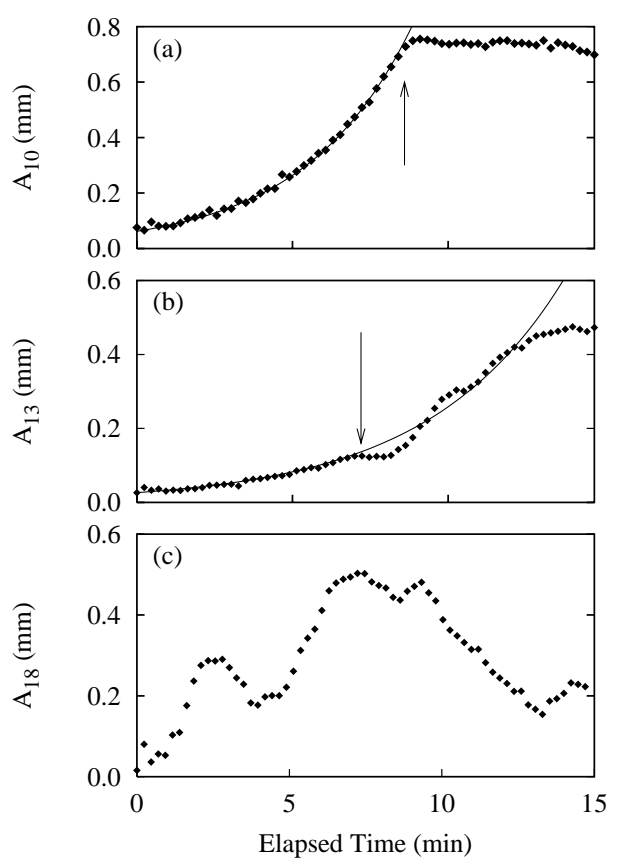

FIG. 4. Three examples for the temporal evolution of the amplitude of a certain mode. The subscripts of each ordinate label denote the mode $m$ in the Fourier decomposition [Eq. (1)]. The arrows indicate the upper boundary for the exponential fits, which are given by the solid lines. Each figure refers to the same value of the shear stress, $\operatorname{Re}_{*}=2.40$.

below). For Figs. 4(a) and 4(b) the amplitude could be well adapted to an exponential growth function of the form $A_{0} \exp (\sigma t)$, and the growth rates $\sigma$ could be extracted. However, this procedure does not work well for Fig. 4(c). This might either be due to a limited temporal resolution or a systematic deviation from exponential growth for modes too far above or below the fastest growing mode. Nevertheless, we find the exponential growth with time for a band of Fourier modes, as was theoretically predicted $[7,18]$, can be experimentally verified for particle Reynolds numbers near threshold, and for modes near the fastest growing mode.

Figure 5 shows the growth rates obtained for five values of the particle Reynolds number. To compare the data to the results of linearized theories, we fitted each set of growth rates for the lower four values of $\mathrm{Re}_{*}$ to a parabola $\sigma(k, \epsilon)=\tau_{0}^{-1}\left[\epsilon-\xi_{0}^{2}\left(k-k_{c}\right)^{2}\right]$, where $\epsilon=\left(\mathrm{Re}_{*}-\mathrm{Re}_{* c}\right) / \mathrm{Re}_{*_{c}}$ and $\mathrm{Re}_{*_{c}}$ is the critical particle Reynolds number for the onset of ripple formation. We found $\operatorname{Re}_{*_{c}}=2.18 \pm 0.04, \quad k_{c}=(0.69 \pm 0.06) \mathrm{cm}^{-1}$, $\tau_{0}=(22 \pm 3) \mathrm{s}$, and $\xi_{0}=(0.16 \pm 0.09) \mathrm{cm}$. The solid lines in Fig. 5 are the result of the fit. The data for $\mathrm{Re}_{*}=2.52$ cannot be fitted using those values. This indicates that for $\epsilon=0.16$ one is outside of the linear regime. The normalized wavelength $\lambda / d=300$ of the fastest growing mode is in line with the measurements given in Ref. [16].

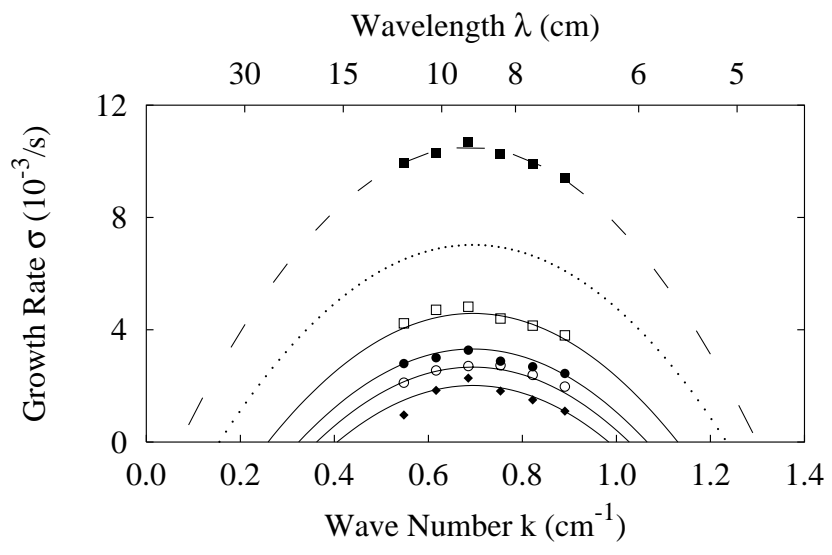

FIG. 5. The growth rates as a function of the wave number for five different values of the particle Reynolds number. On the lower horizontal axis the wave number $k=2 \pi m / L$ is given, on the upper one the corresponding wavelength $\lambda=L / m$. The experiments were performed at a shear stress of $\mathrm{Re}_{*}=2.28$ $(\diamond), 2.30(\bigcirc), 2.34(\bigcirc), 2.40(\square)$, and $2.52(\square)$. The solid lines are parabolic fits to the lower four data sets. The dotted line is the extrapolation for $\operatorname{Re}_{*}=2.52$, while the dashed line indicates an independent parabolic fit to the upper data.

Figure 6 shows the characteristic behavior of the equilibrium state mean amplitude (the plateau in Fig. 3) as a function of $\mathrm{Re}_{*}$. The error bars give the standard deviation of the temporal fluctuations. For the lowest three values of $\mathrm{Re}_{*}$ the mean amplitudes are significantly reduced. Note that these values are in the order of a grain size, as can be seen in Fig. 6 using the right ordinate. An evolution towards a plateau similar to the one shown in Fig. 3 is not observed here; instead we observe irregular fluctuations. In our interpretation these experiments were below the threshold for ripple formation.

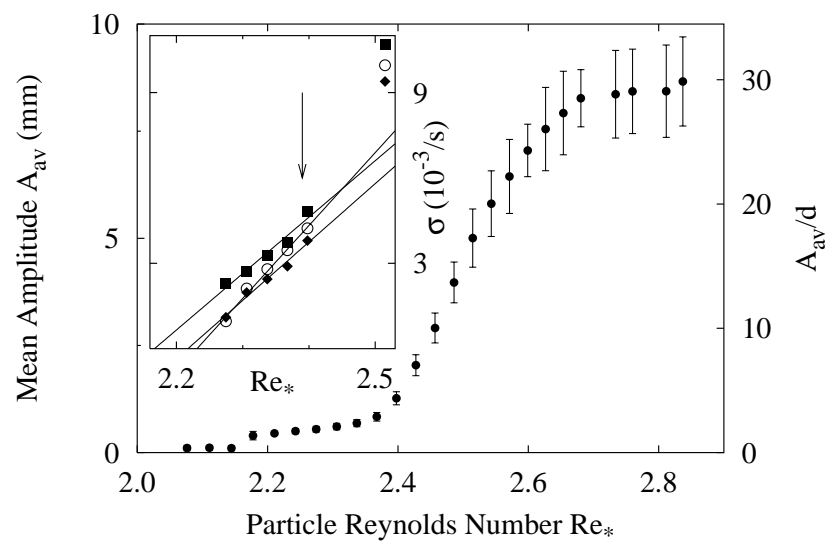

FIG. 6. The mean amplitude (left ordinate in millimeters, right ordinate in units of the grain size) as a function of the shear stress. The error bars denote the standard deviation. The inset shows the behavior of the growth rates for modes $8(\bigcirc)$, $10(\square)$, and $13(\diamond)$. The arrow indicates the upper data points included in the linear fit. 
In the inset of Fig. 6 the growth rates of modes 8,10 , and 13 are given for a subrange 2.1-2.6 in the particle Reynolds number. The solid lines represent linear fits to these growth rates. The point of intersection with the abscissa is interpreted as the instability threshold for the specific Fourier mode. The onset of instability for the fastest growing mode (mode 10 ) is $\mathrm{Re}_{*}=2.17$. Assuming that the lowest three values in Fig. 6 are below the threshold the onset must be in the range $\mathrm{Re}_{*}=2.14-$ 2.18. Based on this agreement between values for the onset determined in two different ways we believe that the initiation of sand ripples can be described in terms of a linearized theory. Additional experiments with different grain sizes indicate that the critical Reynolds number increases linearly with grain diameter. These results will be published elsewhere [19].

The breakdown of a linear description is evident for $\mathrm{Re}_{*}>2.40$ (arrow in the inset of Fig. 6), corresponding to $\epsilon=0.11$. Above this value there is a more pronounced increase in the mean amplitude, an indication that one can no longer neglect nonlinear contributions. From $\mathrm{Re}_{*} \approx$ $2.65(\epsilon \approx 0.22)$ the curve again flattens. This might be caused by the finite height of the channel. Alternatively, this might be the beginning of the restabilization of the unstructured sandbed, which from previous experiments was found to occur for high values of the Reynolds number.

When comparing our results to related studies, it should be noted that both the theoretical predictions of Ref. [7] and the experimental results given in [16] apply to a tiltable channel with an open water surface, and that there is clear evidence that the situation for closed conduits is different from the one observed in open channels [8].

In conclusion, the evolution of sand ripples follows the behavior seen for a wide class of pattern-forming systems. For the early stages in the evolution the ripple amplitude is sufficiently small that single Fourier modes can be treated as noninteracting, which allows a description in terms of linear stability theory. As the ripple amplitude increases one observes nonlinear effects, like saturation of the mean amplitude, "birth" and "death" of ripples, nonmonotonic and correlated evolution of the Fourier modes. These phenomena will be discussed in more detail elsewhere. Thus, despite the striking similarity with better understood pattern-forming systems [3], an approach using a continuum theory is questionable: There must be a crossover for length scales comparable to the grain size.

We thank G. Straßburger, M. Schröter, and M.A. Scherer for experimental help and clarifying discussions. We are grateful for support from Deutsche Forschungsgemeinschaft.

[1] R. A. Bagnold, The Physics of Blown Sand and Desert Dunes (Methuen, London, 1941).

[2] M.S. Yalin, River Mechanics (Pergamon Press, Oxford, England, 1992).

[3] M.C. Cross and P.C. Hohenberg, Rev. Mod. Phys. 65, 851 (1993).

[4] J. F. Kennedy, J. Fluid Mech. 16, 521 (1963).

[5] F. Engelund, J. Fluid. Mech 42, 225 (1970).

[6] J. Fredsøe, J. Fluid Mech. 64, 1 (1974).

[7] K. J. Richards, J. Fluid Mech. 99, 597 (1980).

[8] H. Nakagawa and T. Tsujimoto, J. Hydr. Eng. 110, 467 (1984).

[9] H. Nishimori and N. Ouchi, Phys. Rev. Lett. 71, 197 (1993).

[10] B. T. Werner and D. T. Gillespie, Phys. Rev. Lett. 71, 3230 (1993).

[11] W. Landry and B. T. Werner, Physica (Amsterdam) 77D, 238 (1994).

[12] D. B. Simons, E. V. Richardson, and W. L. Haushild, J. Hydraul. Div. Am. Soc. Civ. Eng. 88, 57 (1962).

[13] G. Parker, J. Hydraul. Div. Am. Soc. Civ. Eng. 101, 211 (1975).

[14] J. H. Baas, Sedimentology 41, 185 (1994).

[15] S. E. Coleman and B. W. Melville, J. Hydr. Eng. 120, 544 (1994).

[16] S. E. Coleman and B. W. Melville, J. Hydr. Eng. 122, 301 (1996).

[17] A. J. Raudkivi, J. Hydr. Eng. 123, 58 (1997).

[18] B. M. Sumer and M. Bakioglu, J. Fluid Mech. 144, 177 (1984).

[19] A. Betat, Ph.D. thesis, Universität Magdeburg, 1999; A. Betat, V. Frette, and I. Rehberg (to be published). 